\title{
CHRONIQUE INTERNATIONALE
}

\section{GOMMUNICATIONS}

Le président passe alors la parole aux auteurs des diverses communications

(10) L'activité géodésique internationale dans le domaine des triangulations.

Mr P. TARDI rend compte en quelques mots des travaux de l'Association Internationale de Géodésie, à l'assemblée Générale de Toronto.

$\left(2^{\circ}\right)$ Au sujet de la réunion de COPENHAGUE de la commission internationale du réseau unifié de nivellement.

Mr G. Descossy fait le point de l'état d'avancement du calcul du R.E.U.N.

$\left(3^{\circ}\right)$ Questions marégraphiques relatives au R.E.U.N.

$\mathrm{Mr}$ L. Cafierre précise les conditions dans lesquelles sont utilisées les indications fournies par les 58 marégraphes et médimarémètres rattachés au R.E.U.N.

(4) Equation générale d'une famille de surfaces de révolution, applicables entre elles, parallèle pour parallèle, dans toute leur étendue.

$\mathrm{Mr}$ Al Clos-Arceduc considère une famille de surfaces de révolution e'équation $r=k f(s)$ où $r$ est le rayon du parallèle, $k$ un paramètre, $s$ l'arc de méridienne, et démontre que ces surfaces de révolution sont applicables entre elles par coïncidence des parallèles correspondant à la même valeur de la variable $s$, arc de méridienne. L'application au choix d'une sphère de bonne approximation pour représenter l'ellipsoïde de référence entre deux parallèles donnés conduit à des formules simples d'application immédiate lorsqu'on des tables de l'ellipsöide considéré.

$\left(^{\circ}\right)$ Discussion sur l'emploi du telluromètre en géodésie.

Aucun point ne restant inscrit à l'ordre du jour, la séance est levée à 18 heures. 